\title{
Aerodynamic continual weft brake
}

\author{
K. Adamek \& P. Karel \\ Textile Machines Research Institute (VUTS), Liberec, Czech Republic
}

\begin{abstract}
This contribution describes the result of a theoretical design for the gentle aerodynamical braking of linear body (weft yarn) transported by air flow. The dynamic model for movement of a discrete mass points system is the main part of the system, the supposed braking force actions result from numerical flow modelling. Theoretical results of such modelling experiments, applied in practice, result in the very expressive production increasing in comparison with the state-of-the-art, together with expressive fabric quality increasing, too.

Keywords: air jet weaving, weft brake, technical cloths, numerical flow modelling.
\end{abstract}

\section{Description of the solved problem}

The air jet weaving is the most productive weaving system, where the weft yarn is transported through the weaving channel by air flow. The system of jet weaving was developed by the foregoer of Textile Machines Research Institute (VUTS) in Liberec - CZ and widespread in the whole world.

In the last time the looms for production of very heavy technical fabrics are developed and realized in VUTS. A standard heavy fabric, as for instance very well known denim for blue jeans, is produced from yarns of a length mass of $100 \mathrm{~g} / \mathrm{km}$. A heavy technical fabric, as for instance woven wall covering, is produced from textured glass yarns of a length mass as far as $600 \mathrm{~g} / \mathrm{km}$.

The standard problem of air jet weaving is remaining the same - in the initial phase of each insertion cycle there is necessary to accelerate the inserted weft yarn. But with increasing machine revolutions and inserted weft yarn mass, too, the new problem arises when the flying weft yarn should be stopped at the end of each pick, first of all by the weaving of very heavy materials.

Example: The loom produces heavy technical fabrics in a weaving width of $2,0 \mathrm{~m}$ and with a weaving frequency of $9 \mathrm{~Hz}$. The weft insertion takes $50 \%$ of 
each period approx., so the average weft insertion velocity reaches of $36 \mathrm{~m} / \mathrm{s}$ $(130 \mathrm{~km} / \mathrm{h})$, the mass of such flying weft length is $1,2 \mathrm{~g}$ and its kinetic energy reaches of $0,78 \mathrm{~J}$. Measured braking action takes about $3 \mathrm{~ms}$, so that the necessary braking power reaches of $260 \mathrm{~W}$ ! Using a standard rash weft stop, the very high and short-term mechanical tension of textile material arises at the end of the insertion period. It is the reason of its permanent deterioration, which is manifested as an inadmissible defect of ready fabric appearance.

\section{Idea of a continual weft brake}

Any mechanical brakes, inserted at the entry into the loom - between the measuring device and the main nozzle - are able to decrease the drag force amplitude to a certain extent, only, because by "gradual" weft velocity decreasing the average picking velocity and loom performance decrease, too. Such a brake cannot have any principal influence on the layout uniformity of permanent deformations along the whole weft length because the braking effect is located in one point, only. The continual weft braking is defined as continuous force acting on each mass element of continuum - the weft yarn - along its whole length.

Solving the above mentioned problem, they were used two independent models - a unique complicated model of weft yarn as a system of individual mass points and standard models of air flow for both weft transport and weft braking.

\subsection{Dynamic model of motion}

The dynamics of the flying weft yarn stopping was described sooner [1]. The one-dimensional system was modelled as the system of individual mass points connected by springs. Solving a relevant system of equations of motion the kinematic values for individual mass points were determined. The consequential more complicated discrete rheologic model [2] contains non-linear springs and dampers between individual mass points. The results received by this method show that the problem solution by this way should be possible in principle and that by suitable selection of continual braking force it should be possible to decrease the peak of the tensile stress in the transported weft during its stopping, without any decreasing of machine performance.

\subsubsection{Weft yarn model}

The used new complex model of weft yarn is described in detail in [3]. The model is composed from $\mathrm{N}$ identical elements ranged in series and connected by rotational kinematic link, see Fig. 1. Necessary weft bending characteristics are defined by rheologic parts concentrated in rotational kinematic pairs. The behaviour of the used springs and dampers is described by constitutional relations, arising from formal validity of linear viscoelasticity.

Tensile characteristics are defined by rheologic parts, their layout is so-called internal structure of element, see Fig. 2. Each element is divided into three 
identical parts, which can be mutually shifted, only. Such internal division of element is realised by reason to differentiate the deformations as elastic and permanent. The relative movement between parts $\mathrm{A}$ and $\mathrm{B}$ of each element describes the permanent deformation. The power link is represented by a friction pair of variable characteristic and by a non-linear damper. The relative movement between parts $\mathrm{B}$ and $\mathrm{C}$ describes the elastic deformation. The power link is represented by a non-linear spring and a non-linear damper.

They were assigned constitutive relations for individual rheologic components of the weft yarn internal structure. Their definition was partially affected by used textile materials, chosen in advance (here textured glass filaments).

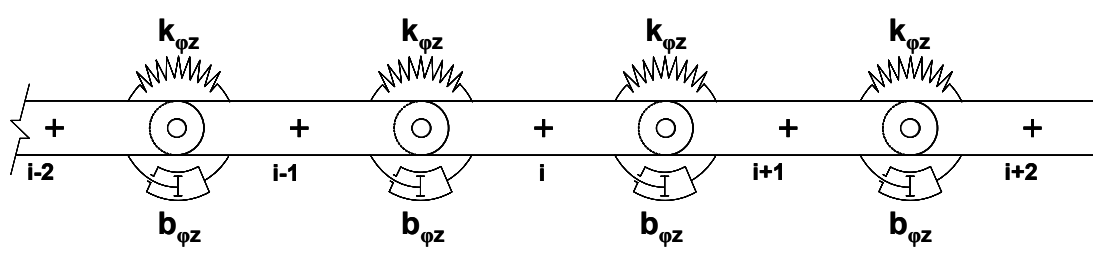

Figure 1: Discrete model of weft yarn.

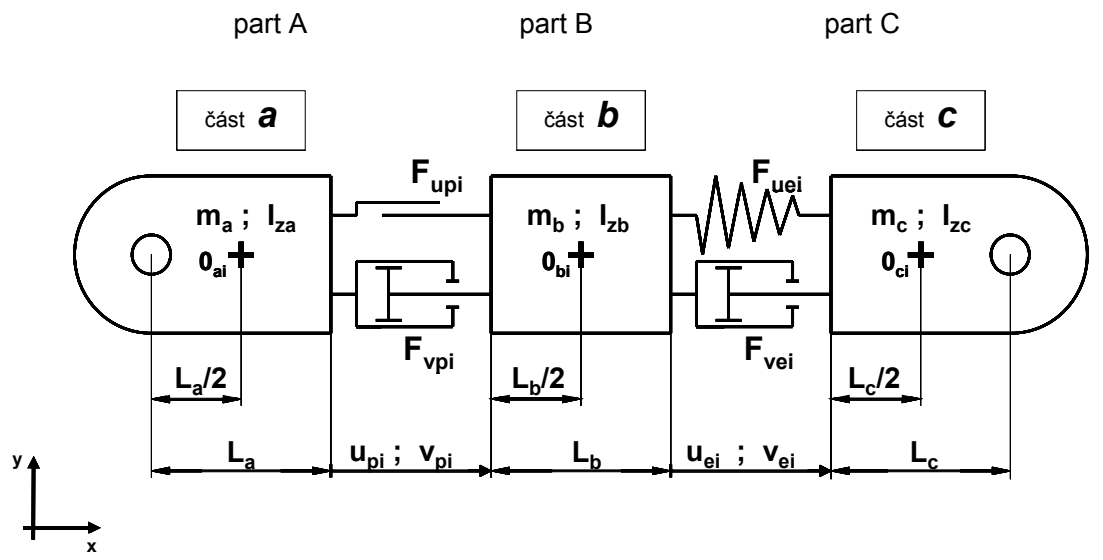

Figure 2: $\quad$ Internal structure of a discrete model element.

The definition of 15 material parameters is quite difficult. Some of them, needed for complicated relations included in [3], were determined on the base of weft material response on static, dynamic and quasidynamic tensile strength tests. Received values depend not only on applied forces, but on the velocity of load application, too.

The complicated field of driving air velocity is defined after Fig. 3. The velocity field is created step by step by both main and tandem nozzles, further by 
periodical effect of a set of auxiliary nozzles and it is finished by a stretch nozzle. The characteristics of used velocity fields from nozzles are defined after standard numerical models of free flows - see for instance [4].

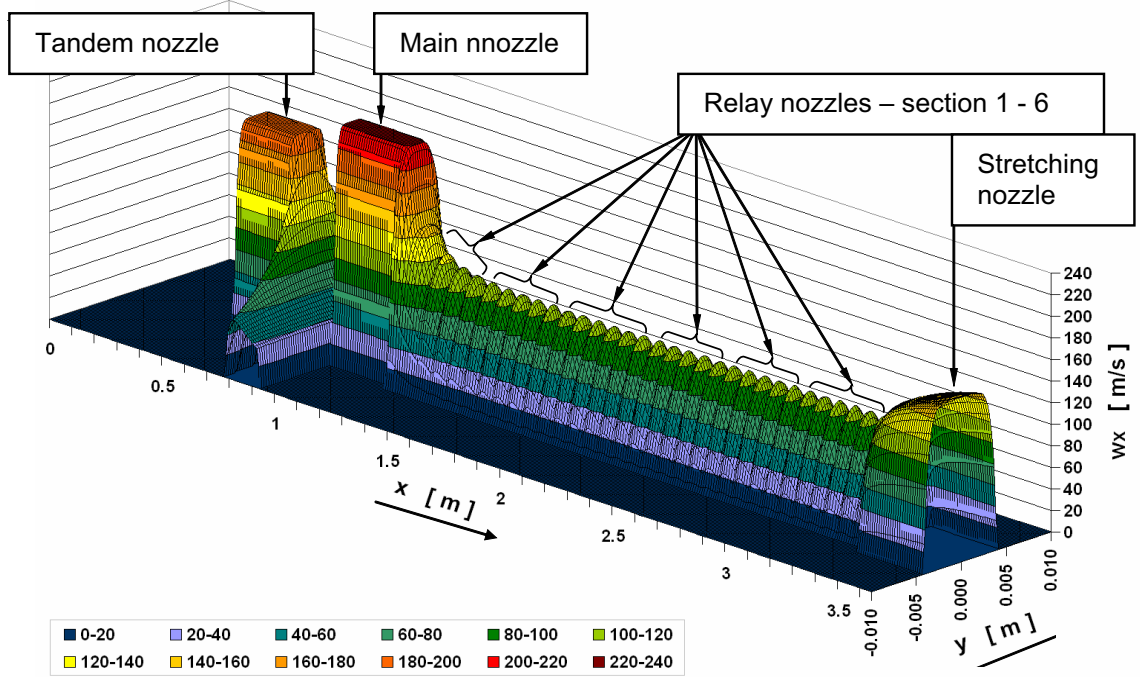

Figure 3: Model of velocity field on air jet loom.

\subsection{Numerical flow model}

The proposed layout of braking nozzles supposes their outlet orientation across to the weaving channel and the location between standard auxiliary weaving nozzles with outlet orientation along the weaving channel.

The next phase of the solution there is the design and realization of a suitable kind of continual braking. First of all it means to design any kind of cross flows with a reasonably even braking effect, which was preliminary designed in such a way to get the relatively easy debugging and solution of complicated models [2, 3]. Initially the examined versions were verified by two-dimensional numerical flow models, followed by 3D numerical flow models.

\subsubsection{Case I}

Individual cross air flows were designed from outlets in a long distance from the weaving channel, approximately $300 \mathrm{~mm}$, to get a relatively even air flow force acting in the channel.

One period of such a continual velocity field is presented in Fig. 4. Beside the natural flow dissipation from the nozzle outlet (situated on the left side) there is simulated the influence of flow passage through the warp yarn system (in the middle) and through the wall of the weaving channel (on the right), too. It is visible that in the area of weaving channel, i.e. just before the wall formed from crosswise oriented thin metal sheets, the velocity field is quite uniform. We can suppose that the braking effect will be quite uniform, too, in conformity with the used dynamic model of motion. 
The device after this numerical flow model was realized as a function model and successfully verified during long-time operation of the air jet loom.

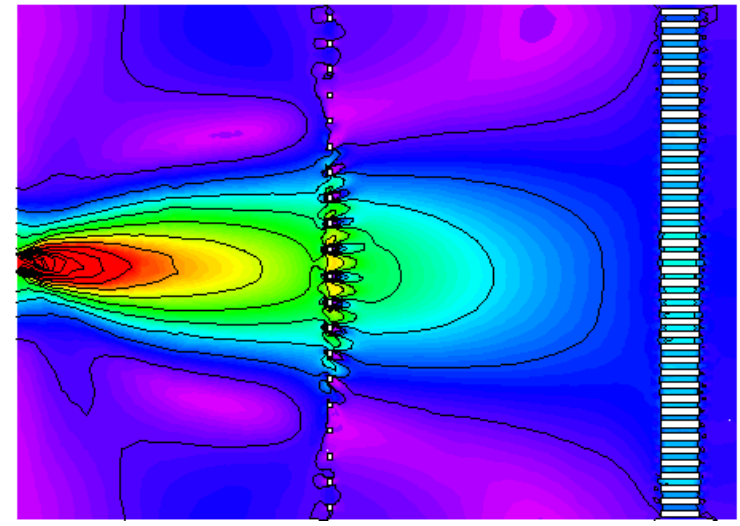

Figure 4: Velocity field from remote nozzle outlet.

\subsubsection{Case II}

Generally, the free flow exiting from the standard nozzle outlet is dissipating in a conical shape. Therefore only a small part of total flow momentum is entering into the narrow picking channel, oriented crosswise to the direction of the braking flow. So the whole system is very uneconomic from the viewpoint of energy efficiency [5]. That is why in the next step of the solution, some shape modifications of nozzle outlet were designed to ensure that the maximum of flow momentum from the nozzle outlet should be evenly spread in the area of a narrow rectangle whose shape corresponds to the longitudinal cross section of the picking channel. Then it should be possible to locate the braking nozzles closer to the channel and to utilize the greatest part of flow momentum together with uniform flow influence along the picking channel. In addition, the whole design installed on the loom is very compact.
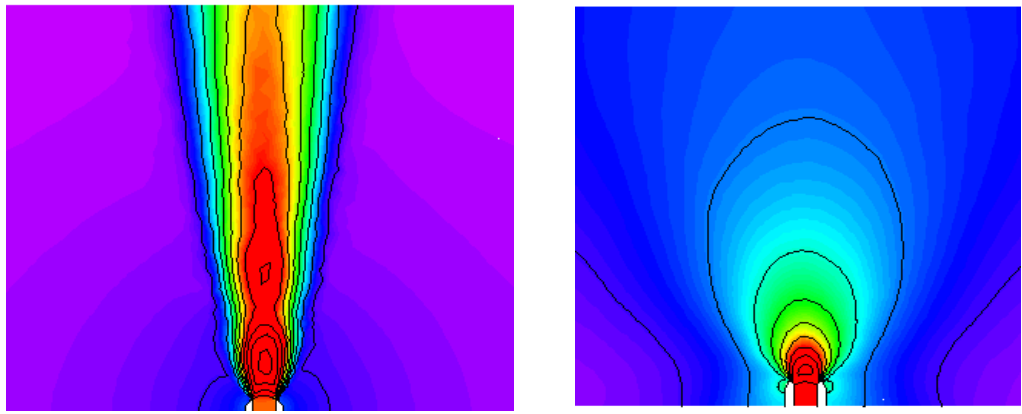

Figure 5: The velocity field from sharp nozzle outlet (left) and from chamfered nozzle outlet (right). 
One of well-known practice for the more intensive free flow dissipation is the increasing of the so-called vorticity coefficient of nozzle by chamfering the outlet internal edge. In Fig. 5 there are mentioned as an illustration two images of free flows from nozzle outlet - with a sharp perpendicular outlet edge where the flow is very pushful (left) and with a chamfered inner edge of the outlet where the flow is fading very quickly (right).

\subsubsection{Nozzle design}

Designing the flat flow from the outlet of a small nozzle, it is not possible to manufacture an inner chamfering of such small dimensions. That is why the nozzle outlet was finally designed as a narrow cut after Fig. 6.

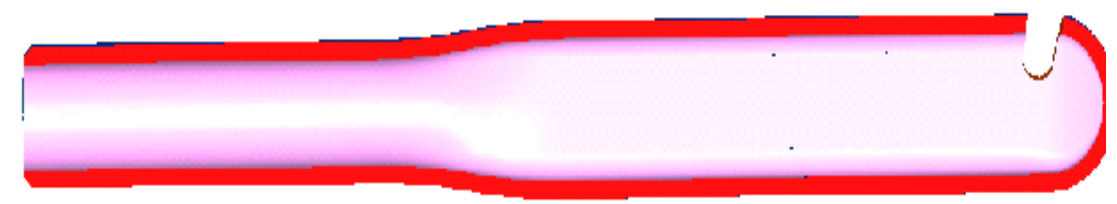

Figure 6: Designed braking nozzle with saw-cut shaped outlet.

The flow modelling in such complicated area is possible as threedimensional, only [6], suitable cross-section shortly after the nozzle outlet see for instance in Fig. 7.

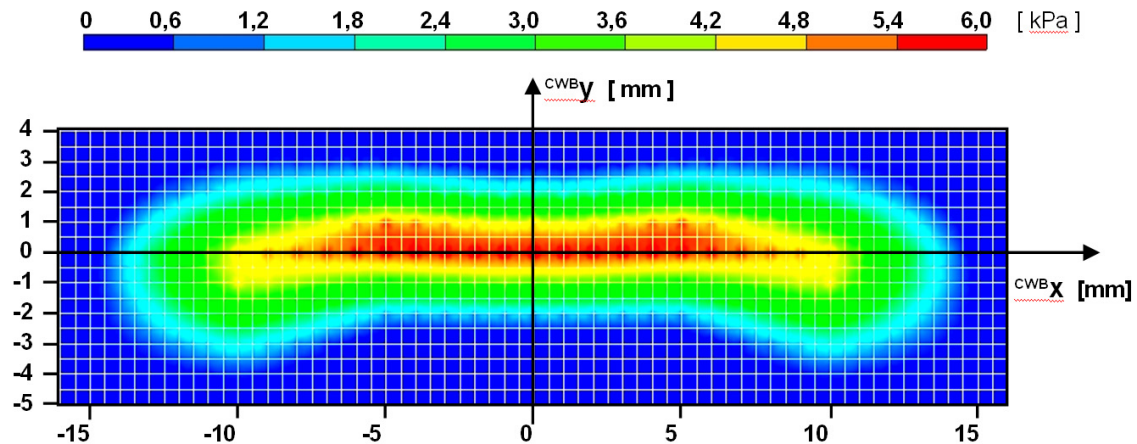

Figure 7: Velocity isolines of flat flow from the nozzle after Fig. 6 (crosssection of 3D model).

The results of numerical modelling were completed by an experiment to verify the extent of the area, really affected by such flow. In next Fig. 8 there is shown the typical measured cross-section through velocity field in the distance of $13 \mathrm{~mm}$ approx. from the slotted outlet in a height of $1 \mathrm{~mm}$ approx. The step of measuring in horizontal direction, i.e. along the picking channel, is here $5 \mathrm{~mm}$, across the channel $1 \mathrm{~mm}$, only. So the area affected by free flow is very flat, with 
dimensions $5 \times 30 \mathrm{~mm}$ approx. and practically the total flow momentum flowing from the nozzle outlet is coming into the picking channel of $6 \mathrm{~mm}$ in width. The influence of its braking effect is effectively enough and at the same time uniform. Comparing both Fig. 7 and Fig. 8 we can state that the coincidence between the 3D numerical model and the real experiment is very good.

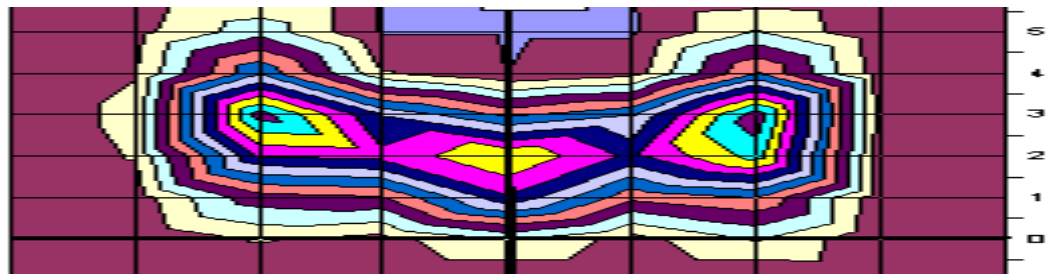

Figure 8: Velocity isolines of flat flow - suitable shape of cross section (measured).

From real experiments it is known that the image quality of flow field is influenced by several parameters (the height, depth, inclination and position of the cut) where some tenths of millimetre only are critical here.

Using the chamfering of the nozzle outlet too small creates the flow similar to a pushful flow after Fig. 5 (left), which is unsuitable for the creation of a uniform and gentle dynamic pressure. On the contrary, the chamfering too large or unsuitably positioned creates an unsuitable flow field, for instance after Fig. 9 where a considerable part of the flow is going out from the $6 \mathrm{~mm}$ width of picking channel as expressive laps. The resulting braking effect is here substantially feebler.

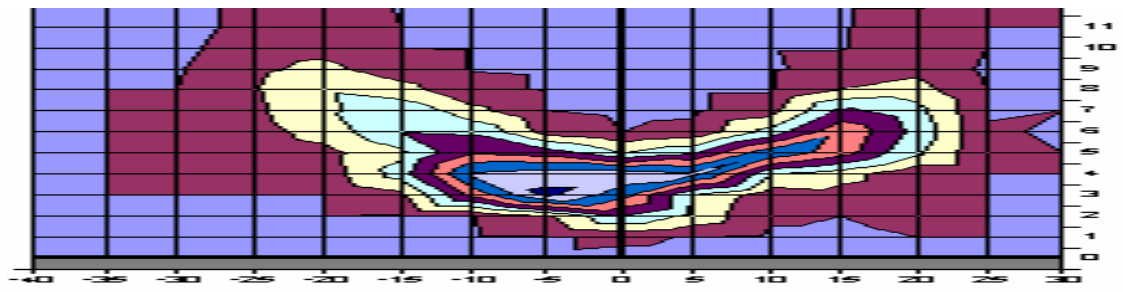

Figure 9: Velocity isolines of flat flow - unsuitable shape of cross section (measured).

Note: Of course, such flat rectangular flow, received from the saw-cut shaped outlet, is naturally transformed in a classical conical shape in the greater distance from the nozzle outlet and finally it is fully dissipating in the undisturbed surroundings. The monitoring of this phenomenon is not the matter of the described solution. 


\section{Discussion of results}

\subsection{Weft model}

The used weft model gives very good coincidence of both modelled and measured behaviour. Fig. 10 shows a time-depending course of tensile force in a very heavy weft material (textured glass 665 tex), both experiment and simulation. On the left-hand side without continual weft braking (CWB), on the right-hand side with short action (2-3 ms) of CWB.
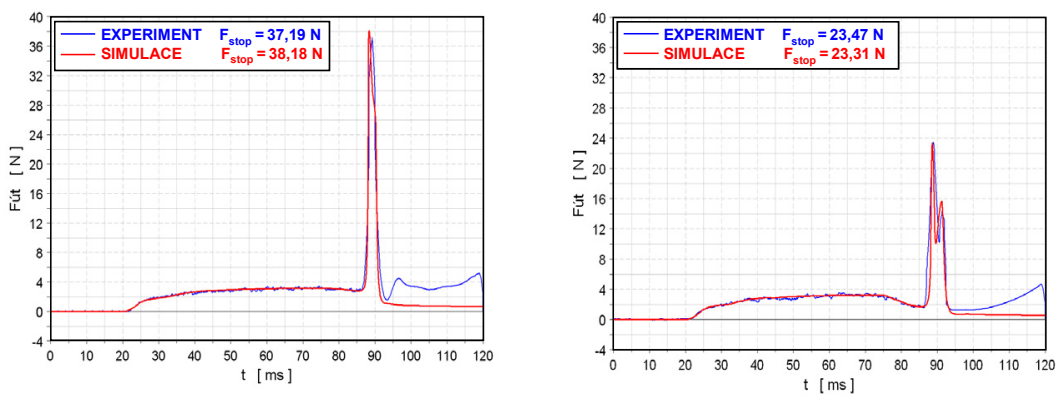

Figure 10: Tensile force - simulation and experiment, without $\mathrm{CWB}$ with CWB.

In both cases the coincidence between experiment and simulation is very good. The difference on the right-hand side of both graphs (after peak value) is influenced by following technological actions, not included in the simulation.

\subsection{Flow model}

The results of the used flow models were used for verification of the initial hypothesis of the solution and further for the seeking of a suitable nozzle outlet shape to get needed flat cross-section of the flow. A 3D numerical model was designed after Fig. 6 in principle. Such modelling replaces hard experiments with many shapes and positions of nozzle outlets when simple outlet adaptations - for instance after Fig. 5 (right) - did not return the expected results.

Note: A next much more complicated model enables to get an image of mutual interaction of such free flow of designed initial deformation with a wall of shaped thin plates, creating the picking channel and situated in the nozzle outlet proximity. Some details about such interaction are presented for instance in [7].

\section{Realisation}

The function model of aerodynamic weft brake after Fig. 4 (case I) has been realised as a supplementary device for the actual loom. Hereto it was necessary to determine several operational parameters of the system (air pressure and 
volume, timing of air flowing, influence of transporting delay between the theoretically programmed valve opening and the real force affect of the flow in the picking channel etc.).

The serial air jet loom was further equipped by an aerodynamic weft brake with nozzles after Fig. 6. After long-term operational trials we can state that the described system, developed on the basis of the above mentioned two theoretical and no-coupled models, operates free and clear. The working frequency of looms equipped by such a device is several times higher than it is usual on other loom types destined for similar materials, further it is possible to weave the materials which cannot be processed on other loom types, in addition with the same or higher fabric quality.

Max. value of weft tensile with CWB is $50 \%$ approx. of the same without CWB - see Fig. 10.

The operation is very reliable; the so-called coefficient of technical exploitation of the loom gets near $100 \%$.

The prototype of the loom was presented at the textile machines exhibition ITMA 2003 in Birmingham and serial machines are in the permanent commercial operation.

For the loom operating at $510 \mathrm{rpm}$, weaving width of $210 \mathrm{~cm}$, is used the very short switching of electromagnetic valve ( $3 \mathrm{~ms}$ approx.) for the air supply to braking nozzles. Corresponding increasing of compressed air consumption takes $20 \%$ approx.

In the same time the excellent fabric quality was reached.

\section{Conclusion}

The presented study combining the dynamic model of movement of a mass points system connected by rheologic links and not-coupled numerical flow modelling validates the initial hypothesis of the solution of the technical problem and makes easier thinking about design execution of the system. The realised system acknowledged the starting theoretical presumptions; the real operation of the loom equipped by such a device is powerful, first-rate, reliable and economical. The complex work [3] gives a good presumption for next development.

\section{References}

[1] Dvorak, J., Placek, B. Weaving process dynamics - the deceleration phase of the weft picking (in Czech). In: Quo vadis textrinum. Proceedings of the 21 st international weaving conference, p. 42-51. NMC Zilina / SK, 1996

[2] Karel, P. Continual weft brake (in Czech). In: Quo vadis textrinum. Proceedings of the 24. international weaving conference, NMC Zilina / SK, 2002. ISBN 80-8565-521-7 
[3] Karel, P. The dynamics of weft movement in final phase of the weft insertion on air let loom. Dissertation thesis. Technical university Liberec, Faculty of machinery, 2006

[4] Adamek, K. Numerical modelling the air flow in parts of air jet loom. In: Comp. Assist. Mech. and Eng. Sciences, 6: 251-261, 1999. Inst. of Fundament. Technol. Research, Polish Academy of Sciences. Special issue containing papers presented at 8th Int. Conf. on Numer. Math. and Comput. Mech. (NMCM98), Miskolc, 1998

[5] Adamek, K. Conditions for high-quality and reliable air jet weft insertion. In: Vlákna a textil 5 (3), 124-130 (1998). ISSN 1335-0617

[6] Zak, J. 3D flow modelling from braking nozzle (in Czech). Report VÚTS Liberec, 2002

[7] Adamek, K. Air flow interaction with porous wall (in Czech). In: Topical problems of fluid mechanics 2001. Inst. of Thermomech. Czech Academy of Sciences, Prague, 2001. ISBN 80-85918-62-5 